\title{
Fast-Atom Bombardment Mass Spectrometry and Low Energy Collision-Induced Tandem Mass Spectrometry of Tauroconjugated Bile Acid Anions
}

\author{
Vincent Stroobant and Edmond de Hoffmann \\ Service de Spectrométrie de Masse, Université Catholique de Louvain, Louvain-la-Neuve, Belgium
}

Raymond Libert and François Van Hoof

Laboratoire des Maladies Métaboliques, Cliniques St. Luc, Brussels, Belgium

Fragmentation of negative ions produced by fast-atom bombardment (FAB) from 14 tauroconjugated bile acids and some of their deuterated analogs has been studied by mass spectrometry and by collision-induced dissociation (CID) tandem mass spectrometry at low energy.

Low energy collision-induced dissociation of the deprotonated molecules $[\mathrm{M}-\mathrm{H}]^{-}$of these tauroconjugated bile acids leads to both charge-driven and charge-remote fragmentations (CRF). The former yields neutral loss from the side chain with charge migration during the fragmentation process. These fragments dominate the CID spectra, but are absent from the FAB spectra. Their relative abundances are dependent on the number and the positions of the hydroxyl groups in the steroid nucleus and thus permit distinction among some positional isomers.

The CRF fragments correspond to cleavages in the side chain up to fragmentations across the steroid rings with charge retention on the sulfonate group. These CRF fragments, which also are useful for structural identification, are less intense in CID than in FAB spectra. It appears that these charge-remote fragments are favored by unsaturation in the steroid rings, either as keto groups or as endocyclic double bonds. Tandem mass spectrometry combined with the use of deuterated analogs demonstrates that the structures of the survivor pseudomolecular ions and of the CRF fragments are not rearranged. (/ Am Soc Mass Spectrom 1995, 6, 588-596)

$\mathrm{F}$ ast-atom bombardment (FAB) ionization combined with tandem mass spectrometry is very useful in the qualitative analysis of bile acids, free or conjugated. It not only yields information on the structure of isolated bile acids, but allows fast analysis of complex bile acid mixtures after a short workup from biological samples ([1-7] and Libert, R.; Van Hoof, F.; Stroobant, V.; de Hoffmann, E., unpublished).

Charge-remote fragmentations are gas-phase decompositions of closed-shell ions that occur at sites that are physically remote from the location of the charge, which is not involved in the reaction $[8,9]$. These fragmentations may be observed at low and high collision energies (few tens of electronvolts and a few kiloelectronvolts, respectively), but often appear to be favored at high energy [10].

Address reprint requests to Vincent Stroobant, Spectrométrie de Masse, Place Pasteur 1, 1348 Louvain-la-Neuve, Belgium.
At high collision energy, the deprotonated molecules $[\mathrm{M}-\mathrm{H}]^{-}$of free or conjugated bile acids predominantly yield fragment ions that arise by reactions that occur remote from the charge. These reactions generate fragments from different positions in the side chain and across the rings of the steroid framework [1-3]. By a comparison of various cholestane derivatives, it has been observed that charge-remote fragmentation (CRF) bile acid ring cleavage preferentially occurs at the position most distant from the charge site. The location of the ring substituents can be determined from the mass shifts of these fragments. Some information about the stereochemistry of the $A / B$ ring junction can be obtained from the relative abundances of ring-cleavage fragments [2]. Similar information can be obtained from the sodium adducts $[\mathrm{M}-\mathrm{H}+\mathrm{Na}+\mathrm{Na}]^{+}$of the bile acids by using positive ion FAB tandem mass spectrometry at high energy $[4,5]$.

In previous articles we have shown the usefulness of low collision energy FAB-MS/MS with a triple 
quadrupole instrument in the diagnosis of metabolic disorders by analysis of underivatized bile acids, conjugated or not, in urine and in plasma $([6,7]$ and Libert, R.; Van Hoof, F.; Stroobant, V.; de Hoffmann, E., unpublished). These analytical possibilities have been applied usefully to several hundred biological samples. However, several questions about the fragmentation of these bile acid ions, in the source or in the low energy collision cell, remained unresolved. The mechanisms of many fragmentations were not established: Were some of them charge-remote fragmentations or the products of charge-driven fragmentations? Did they oridinate directly from the $[\mathrm{M}-\mathrm{H}]^{-}$anion or from a stepwise fragmentation? Was the original structure present in some fragments or did they result from rearrangement? In this article we address these questions in a systematic study of 14 tauroconjugated bile acids and some of their deuterated analogs. We report on progress in understanding their fragmentation. In addition to fundamental interest, this new insight into the fragmentation pathways is useful in the interpretation of spectra of unknown compounds in biological samples.

\section{Experimental}

\section{Materials}

Free acids of hyocholic, apocholic, $\Delta 5$ cholenoic acids, and tauroconjugates of lithocholic, chenocholic, ursodeoxycholic, deoxycholic and cholic acids were purchased from Sigma Chemical Co. (St. Louis, MO). Free acids of murocholic and $3 \beta$-hydroxycholic acids and tauroconjugates of cholanic, hyodeoxycholic, and dehydrocholic acids were obtained from Steraloïds Inc. (Wilton, NH).

The syntheses of taurine and the 3-amino-1propanesulfonic acid conjugates of the free bile acids were performed as described in detail by Zhang et al. [11]. In short, one equivalent of a bile acid was reacted with 10 equivalents of taurine (Sigma Chemical Co.) or 10 equivalents of 3-amino-1-propanesulfonic acid (Janssen Chimica, Geel, Belgium) and 10 equivalents of 1-ethyl-3-(3-dimethylaminopropyl) carbodiimide hydrochloride (Janssen Chimica). After $24 \mathrm{~h}$ the tauroconjugated bile acids were purified by passing the reaction solution through a SEP-PAK ${ }^{\oplus}$ cartridge of octadecylsilane-bonded silica (Waters-Millipore Co., Milford, MI). After washing with water, the bile acid was eluted with methanol.

Bile acids labeled by deuterium exchange in the hydroxyl and in the amide groups were prepared by dissolving these bile acids in MeOD and mixture of the solution with glycerol- $d_{3}$ (Aldrich Chemical Co., Milwaukee, WI) as matrix.

The taurocholic-3,7,12- $d_{3}$ acid was synthesized in ethanol by reduction of 1 equivalent of taurodehydrocholanic acid with 10 equivalents of sodium borodeuteride (Merck, Darmstadt, Germany) at room tempera- ture. After $2 \mathrm{~h}$ the reaction mixture was diluted with water and passed through a SEP-PAK cartridge as described in the preceding text.

\section{Mass Spectrometry}

Mass spectrometric measurements were performed with a Finnigan-MAT TSQ 70 mass. spectrometer (San Jose, CA). An Ion Tech (Middlesex, UK) FAB gun gave a primary beam of xenon at $8 \mathrm{kV}$ and $0.6 \mathrm{~mA}$. The collision-induced dissociation (CID) spectra were obtained by using xenon as the collision gas at 0.7 mtorr (uncorrected gauge reading pressure) in the rf-only collision cell. All of the CID spectra used were acquired with a collision offset voltages of $10 \mathrm{~V}$.

Samples were dissolved in methanol $(2 \mathrm{mg} / \mathrm{mL})$, and $1 \mu \mathrm{L}$ of this solution was deposited in a drop of glycerol on the tip of the FAB probe.

\section{Results and Discussion}

\section{Fast-Atom Bombardment Mass Spectrometry}

A series of 14 tauroconjugated bile acids was investigated in the negative ion mode by fast-atom bombardment and tandem mass spectrometry at low collision energy. The structures of the conjugated bile acids examined in this study are shown in Table 1.

The negative ion FAB spectra of all the conjugated bile acids studied are dominated by two intense peaks that correspond to the deprotonated molecule [M $\mathrm{H}]^{-}$and the sodium adduct ion $[\mathrm{M}-2 \mathrm{H}+\mathrm{Na}]^{-}$. These spectra also display low abundance fragments. This general trend is illustrated in Figure 1 by the negative ion FAB spectrum of taurolithocholic acid (2).

The negative ion FAB spectra of all these tauroconjugated bile acids have common fragmentation pathways that will now be discussed. To our knowledge this is the first detailed description of the negative ion FAB spectra of the tauroconjugated bile acids.

Under our experimental conditions, $[\mathrm{M}-\mathrm{H}-2]^{-}$, $[\mathrm{M}-\mathrm{H}-16]^{-}$, and $[\mathrm{M}-\mathrm{H}-18]^{-}$ions always are observed in these spectra. These ions could correspond to the loss of hydrogen, methane, and water molecules from the $[\mathrm{M}-\mathrm{H}]^{-}$ion.

A series of ions at $m / z 80,94,107,124,150,151$, $165,178,206,232,234,246,248$, and 288 that results from fragmentations of the side chain and cleavage across the $C$ and $D$ rings, also is always present (fragmentations $\mathrm{C}$ to $\mathrm{L}$ in Scheme $\mathrm{I}, n=1$ ). These assignments are confirmed by the fragmentation of the taurocholanic- $d_{1}$ acid $\left(1-\mathrm{d}_{1}\right)$, prepared by deuterium exchange of the amide hydrogen, and of the 3aminopropanesulfonate (AMPS) conjugate of cholanic acid, a compound homologous to taurocholanic acid (Scheme I, $n=2$ ). Indeed, as. shown in Table 2, the fragment ions $C$ to I (Scheme I) are shifted by $1 \mathrm{u}$ in the FAB spectrum of the taurocholanic- $d_{1}$ acid $\left(1-d_{1}\right)$ and by $14 \mathrm{u}$ in the FAB spectrum of the 3-aminopro- 
Table 1. Structure and nomenclature of the tauroconjugated bile acids investigated

\begin{tabular}{|c|c|c|c|c|c|c|c|}
\hline $\mathrm{N}^{\circ}$ & Trivial name & Chemical name & $R_{1}$ & $\mathbf{R}_{2}$ & $\mathbf{R}_{\mathbf{3}}$ & $\mathbf{R}_{4}$ & {$[M-H]$} \\
\hline 1 & Taurocholanic acid & $5 \beta$-cholanic acid & $\mathrm{H}$ & $\mathrm{H}$ & $H$ & $\mathrm{H}$ & $466^{\mathrm{a}}$ \\
\hline 2 & Taurolithocholic acid & $\begin{array}{l}N \text {-(2-sulfoethyl)-amide } \\
5 \beta \text {-cholanic acid-3 } \alpha \text {-ol } \\
N \text {-(2-sulfoethyl)-amide }\end{array}$ & $\alpha \mathrm{OH}$ & $\mathrm{H}$ & $\mathrm{H}$ & $\mathrm{H}$ & 482 \\
\hline 3 & Tauro- $3 \beta$-hydroxychoanic acid & $\begin{array}{l}5 \beta \text {-cholanic acid-3 } \beta \text {-ol } \\
N \text {-(2-sulfoethy } 1) \text {-amide }\end{array}$ & $\beta \mathrm{OH}$ & $H$ & $\mathrm{H}$ & $\mathrm{H}$ & 482 \\
\hline 4 & Taurohyodeoxycholic acid & $\begin{array}{l}5 \beta \text {-cholanic acid-3 } \alpha, 6 \alpha \text {-diol } \\
N \text {-(2-sulfoethyl)-amide }\end{array}$ & $\alpha \mathrm{OH}$ & $\alpha \mathrm{OH}$ & $\mathrm{H}$ & $\mathbf{H}$ & 498 \\
\hline 5 & Tauromurocholic acid & $\begin{array}{l}5 \beta \text {-cholanic acid-3 } \alpha, 6 \beta \text {-diol } \\
N \text {-(2-sulfoethyl)-amide }\end{array}$ & $\alpha \mathrm{OH}$ & $\beta \mathrm{OH}$ & $\mathrm{H}$ & H & 498 \\
\hline 6 & Taurochenodeoxycholic acid & $\begin{array}{l}5 \beta \text {-cholanic acid-3 } \alpha, 7 \beta \text {-diol } \\
N \text {-(2-sulfoethyl)-amide }\end{array}$ & $\alpha \mathrm{OH}$ & $\mathrm{H}$ & $\alpha \mathrm{OH}$ & H & 498 \\
\hline 7 & Tauroursodeoxycholic acid & $\begin{array}{l}5 \beta \text {-cholanic acid-3 } \alpha, 7 \beta \text {-diol } \\
N \text {-(2sulfoethyl)-amide }\end{array}$ & $\alpha \mathrm{OH}$ & $H$ & $\beta \mathrm{OH}$ & $\mathrm{H}$ & 498 \\
\hline 8 & Taurodeoxycholic acid & $\begin{array}{l}5 \beta \text {-cholanic acid-3 } \alpha, 12 \alpha \text {-diol } \\
N \text {-(2-sulfoethyl)-amide }\end{array}$ & $\alpha \mathrm{OH}$ & H & $H$ & $\alpha \mathrm{OH}$ & 498 \\
\hline 9 & Taurocholic acid & $\begin{array}{l}5 \beta \text {-cholanic acid-3 } \alpha, 7 \alpha, 12 \alpha \text {-triol } \\
N \text {-(2-sulfoethyl)-amide }\end{array}$ & $\alpha \mathrm{OH}$ & $\mathrm{H}$ & $\alpha \mathrm{OH}$ & $\alpha \mathrm{OH}$ & 514 \\
\hline 10 & Taurohyocholic acid & $\begin{array}{l}5 \beta \text {-cholanic acid-3 } \alpha, 6 \alpha, 7 \alpha \text {-triol } \\
N \text {-(2-sulfoethyl)-amide }\end{array}$ & $\alpha \mathrm{OH}$ & $\alpha \mathrm{OH}$ & $\alpha \mathrm{OH}$ & $\mathrm{H}$ & 514 \\
\hline 11 & Taurodehydrocholic acid & $\begin{array}{l}5 \beta \text {-cholanic acid-3,7,12-trione } \\
N \text {-(2-sulfoethyl)-amide }\end{array}$ & 0 & $\mathrm{H}$ & 0 & 0 & 508 \\
\hline 12 & Tauro-2-cholenic acid & $\begin{array}{l}\text { 2,(5B)-cholenic acid } \\
N \text {-(2-sulfoethyl)-amide }\end{array}$ & $\mathbf{H}$ & $\mathrm{H}$ & $\mathrm{H}$ & $\mathrm{H}$ & 464 \\
\hline 13 & $\begin{array}{l}\text { Tauro- } 3 \beta \text {-hydroxy- } \\
5 \text {-cholenic acid }\end{array}$ & $\begin{array}{l}5 \text {-cholenic acid-3 } \beta \text {-ol } \\
N \text {-(2-sulfoethyl)-amide }\end{array}$ & $\beta \mathrm{OH}$ & $\mathrm{H}$ & $\mathrm{H}$ & $\mathrm{H}$ & 480 \\
\hline 14 & Tauroapocholanic acid & $\begin{array}{l}8(14),(5 \beta) \text {-cholenic acid-3 } \alpha, \\
12 \alpha \text {-diol } \\
N \text {-(2-sulfoethyl)-amide }\end{array}$ & $\alpha \mathrm{OH}$ & $\mathrm{H}$ & $H$ & $\alpha \mathrm{OH}$ & 496 \\
\hline
\end{tabular}

Mass-to-charge ratios of the deprotonated molecules.

panesulfonate conjugate of cholanic acid. Some of these fragments also are present in the low energy CID spectra discussed in succeeding text.

The FAB spectra of tauroconjugated bile acids also display two fragmentations that lead to ions that strongly reflect the structure differences among the various tauroconjugated bile acids studied. Here, because the tauroconjugated bile acids differ only in the nature and the location of substituents on the steroid ring system, these fragment ions have been interpreted without ambiguity by comparison of the spectra of different bile acids. The first of these two fragmentations (B in Scheme I) leads to ions at $m / z 342,358$, or 356 dependent on the presence or absence of a hydroxyl or a keto group at the 12 position. Because the masses of these ions are not influenced by the presence of either deuterium labeling or different substituents in the $A$ and $B$ rings, they must result from a chargeremote fragmentation that corresponds to a cleavage of the $B$ ring across the 7-8 and 9-10 bonds (fragmentation $B$ in Scheme $I, n=1$ ). The second of these charge-remote fragmentations ( $\mathrm{A}$ in Scheme $\mathrm{I}$ ) yields 


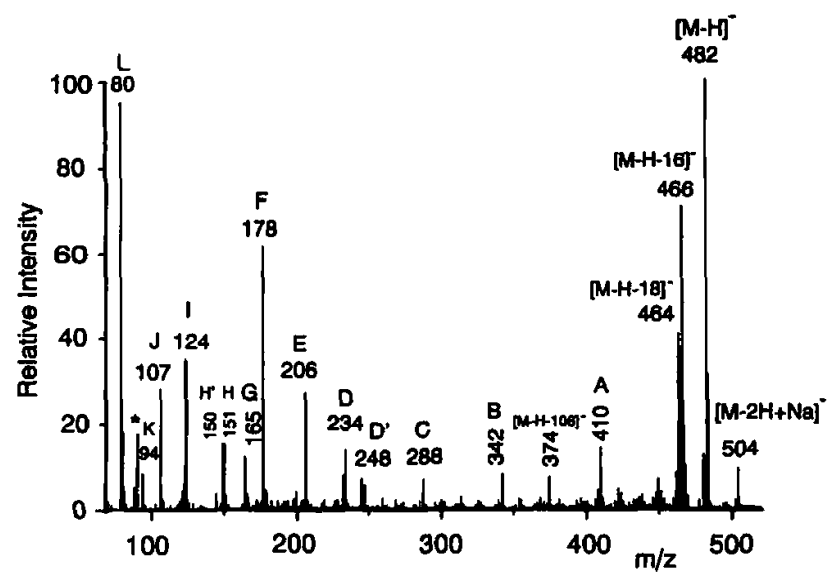

Figure 1. Negative ion FAB mass spectrum of the taurolithocholic acid (2). The intensities of ions between $m / z 70$ and 470 have been magnified by a factor of 10 . The peak labeled $*$ is from the glycerol matrix. The capital letters characterize ions represented in the fragmentation pattern shown in Scheme I.

ions at masses between $m / z 410$ and 442, dependent on the presence of hydroxyl or keto groups at the 6,7 , or 12 positions. Because the masses of these fragment ions are not influenced by the presence of either deuterium labeling or a different substituent in the $A$ ring, they correspond to cleavages of this ring across the 1-10 and 4-5 bonds (fragmentation $A$ in Scheme $I$, $n=1)$. They correspond to $[\mathrm{M}-\mathrm{H}-56]^{-},[\mathrm{M}-\mathrm{H}-$ $72]^{-}$and $[\mathrm{M}-\mathrm{H}-70]^{-}$, dependent on the presence or absence of a hydroxyl or keto group at the 3 position. As summarized in Table 3, the identities of the bonds broken in these two fragmentation pathways are clearly demonstrated by both the deuterium labeling and comparison of the spectra from differently substituted bile acids. As will be shown in succeeding text, these fragmentation pathways also are observed in the low energy CID fragmentation spectra.

The last common fragmentation in the FAB spectra of the tauroconjugated bile acids studied in the present work corresponds to a loss of $108 \mathrm{u}$. Indeed the [M $\mathrm{H}-108]^{-}$ion always is present, whatever the substitution or the deuterium labeling. Thus, this loss can hardly come from the steroid nucleus. In the spectrum of the 3-aminopropanesulfonate conjugate of cholanic

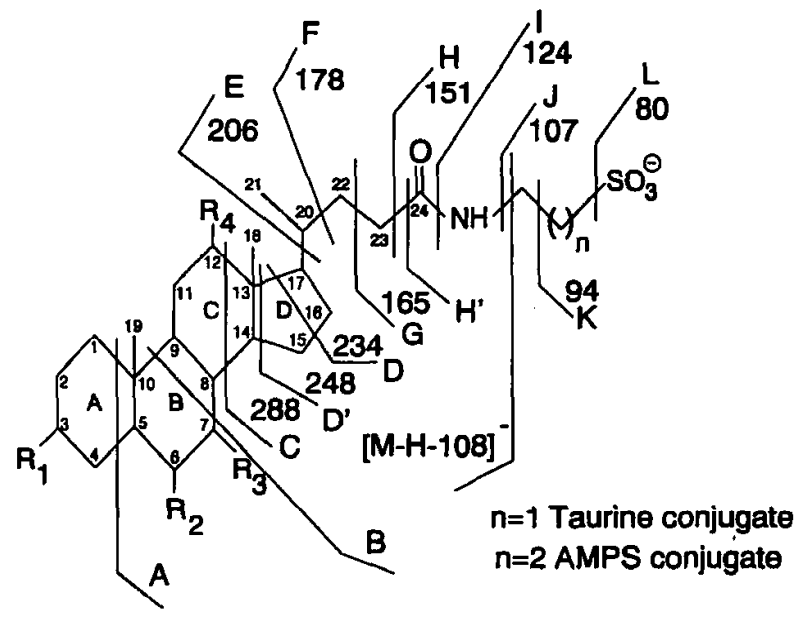

Scheme 1. Fragmentation pattern of the tauroconjugated bile acids observed in FAB mass spectrometry. $R_{1}, R_{2}, R_{3}, R_{4}$ correspond to a hydrogen atom, a hydroxyl group, or a keto group as described in Table 1.

acid, the observed loss becomes $122 \mathrm{u}$ instead of $108 \mathrm{u}$ and indicates that the neutral lost contains a part of the taurine moiety. Furthermore, a scan of the precursors of this $[\mathrm{M}-\mathrm{H}-108]^{-}$ion does yield signals at $[\mathrm{M}-$ $\mathrm{H}-16]^{-}$and $[\mathrm{M}-\mathrm{H}]^{-}$(spectrum not shown). After these data are combined, the sole possibility is the loss from the $[\mathrm{M}-\mathrm{H}]^{-}$of a $\mathrm{CH}_{2} \mathrm{CH}_{2} \mathrm{SO}_{3}$ neutral species, which corresponds to a part of the taurine moiety, which occurs in two steps: the loss of an oxygen atom from the sulfonate group ( $16 \mathrm{u}$ ) followed by a loss of $\mathrm{C}_{2} \mathrm{H}_{4} \mathrm{SO}_{2}(92 \mathrm{u})$. The loss of an oxygen atom from the sulfonate group could seem odd, but is confirmed by the presence of a peak at $[\mathrm{M}-\mathrm{H}-16]^{-}$in the negative ion FAB spectra of other molecules that contain a sulfonate group like taurine, acetyltaurine, or benzenesulfonic acid (spectra not shown). This loss may be attributed either to the tauroconjugated bile acid reduced in the FAB matrix prior to the ionization or to the fragmentation of the pseudomolecular ion in the gas phase.

The FAB mass spectrometry fragmentation pathways of the tauroconjugated bile acids are summarized in Scheme I. Comparison between these FAB spectra and high energy CID spectra of the tauroconju-

Table 2. Mass-to-charge ratio of the fragments from the side chain of the tauroconjugate of cholanic acid (1) and of the corresponding fragments of its deuterium-labeled compound and of its aminopropanesulfonate (AMPS) homologous conjugate

\begin{tabular}{|c|c|c|c|c|c|c|c|c|c|c|}
\hline \multirow[b]{2}{*}{ Bile acids } & \multicolumn{10}{|c|}{ Fragments $^{\theta}$} \\
\hline & $J$ & 1 & $\mathrm{H}^{\prime}$ & $H$ & $\mathbf{G}$ & $F$ & $E$ & D & $\mathrm{D}^{\prime}$ & C \\
\hline Taurocholanic (1) & 107 & 124 & 150 & 151 & 165 & 178 & 206 & 234 & 248 & 288 \\
\hline Taurocholanic- $d_{1}\left(1-d_{1}\right)$ & 107 & 125 & 151 & 152 & 166 & 179 & 207 & 235 & 249 & 289 \\
\hline $\begin{array}{l}\text { AMPS conjugate of } \\
\text { cholanic acid }\end{array}$ & 121 & 138 & 164 & 165 & 179 & 192 & 220 & 248 & 262 & 302 \\
\hline
\end{tabular}

${ }^{a}$ The capital letters characterize each of the ions represented in the fragmentation pattern shown in Scheme I. 
Table 3. $A$ and $B$ steroid ring fragmentations that occur by $C R F$

\begin{tabular}{|c|c|c|c|}
\hline Bile acids & $\begin{array}{l}\text { Position of the } \\
\text { substituent } \\
\text { groups }\end{array}$ & $\begin{array}{l}\text { Masses of the fragment } \\
\text { ions that correspond to } \\
\text { cleavage of the } A \text { ring }\end{array}$ & $\begin{array}{l}\text { Masses of the fragment } \\
\text { ions that correspond to } \\
\text { cleavage of the } B \text { ring }\end{array}$ \\
\hline Taurocholanic acid (1) & - & 410 & 342 \\
\hline Taurolithocholic acid (2) & $3 \mathrm{OH}$ & 410 & 342 \\
\hline Taurohyodeoxycholic acid (4) & $3,6 \mathrm{di} \mathrm{OH}$ & 426 & 342 \\
\hline Taurochenodeoxycholic acid (6) & $3,7 \mathrm{di} \mathrm{OH}$ & 426 & 342 \\
\hline Taurodeoxycholic acid (8) & $3,12 \mathrm{di} \mathrm{OH}$ & 426 & 358 \\
\hline Taurocholic acid (9) & $3,7,12$ tri OH & 442 & 358 \\
\hline Taurocholic acid $\left(9-d_{4}\right)^{8}$ & $3,7,12$ tri OD & 445 & 360 \\
\hline Taurocholic-3,7,12-d $d_{3}$ acid $^{b}$ & $3,7,12$ tri OH & 444 & 359 \\
\hline Taurohyocholic acid $(10)$ & $3,6,7$ tri OH & 442 & 342 \\
\hline Taurodehydrocholic acid (11) & $3,7,12$ tri keto & 438 & 356 \\
\hline
\end{tabular}

"Tauroconjugated bile acid labeled by deuterium exchange in the hydroxyl groups and the amide group.

${ }^{6}$ Tauroconjugated bile acid labeled by reduction with deuteride in the 3,7 , and 12 positions.

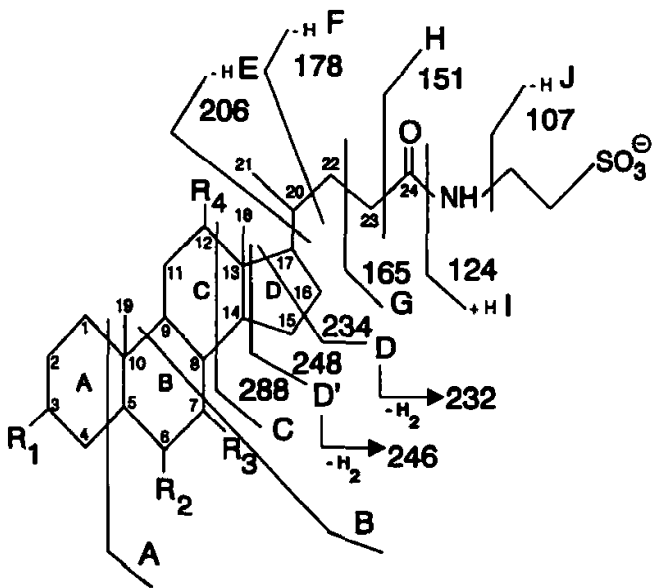

Scheme II. Series of fragment ions with charge retention on the sulfonate group observed in FAB CID tandem mass spectrometry at low energy of the tauroconjugated bile acids. $R_{1}, R_{2}, R_{3}, R_{4}$ correspond to a hydrogen atom, a hydroxyl group, or a keto group as described in Table 1.

gated bile acid anions $[\mathrm{M}-\mathrm{H}]^{-}$presented in the literature $[1,3]$ shows that they display similar patterns in the middle and high mass regions. Comparison in the low mass region (below $m / z 200$ ) is not possible because the high energy CID spectra in the literature do not display this region.

\section{Fast-Atom Bombardment Collision-Induced Tandem Mass Spectrometry of $\left[\mathrm{M}-\mathrm{H}^{-}\right.$at Low Energy}

The FAB CID tandem mass spectra of the $[\mathrm{M}-\mathrm{H}]^{-}$ ions of the 14 tauroconjugated bile acids studied here have been obtained. Typical spectra are shown in Figure 2. Two important series of peaks have been observed.

The first series corresponds to the fragments $A$ to $J$ in Scheme II with charge retention on the sulfonate group. The lower mass ions at $m / z 107,124,151,165$, 178 , and 206 result from fragmentations of the side chain that occurs by homolytic cleavage or with hydrogen atom migration. The higher mass ions that corre- spond to fragments A to D in Scheme II result from cleavages across the steroid ring system. Part of them appear at fixed masses $m / z 232,234,246,248$, and 288 that correspond to cleavages of the $C$ and $D$ rings (fragmentations $C, D^{\prime}$, and $D$ in Scheme II), whereas the masses of the others ions depend on the presence or absence of substituents on the $A$ and $B$ rings (fragmentations A and B in Scheme II). The latter allow the determination of the nature and location of some substituents on the steroid ring system as well as the presence of endocyclic unsaturation. All the ions of this first series (A to $J$ in Scheme II) overlap those observed in the FAB spectrum, except for ions at $m / z$ 80,94 , and 150 , which are present only in the FAB spectrum.

Fragmentations of the side chain ( $\mathrm{E}$ to $\mathrm{J}$ in Scheme II) can occur by homolytic cleavage or by hydrogen atom migration. Fragment ions formed by homolytic cleavage appear at $m / z 80\left(\mathrm{SO}_{3}^{--}\right), 94\left(\mathrm{CH}_{2} \mathrm{SO}_{3}^{-}\right), 151$, and 165 (fragmentations $G$ and $H$ in Scheme II). All of these radical anions are stabilized by delocalization of the unpaired electron with the sulfonate or the amide groups. Formation of the other ions derived from the side chain involve a hydrogen migration from the fragment ion to the neutrals, with the exception of the ion at $m / z 124$, for which the migration occurs in the opposite direction.

Although it is reasonable to assume that the sulfonate group carries the negative charge in the [M $\mathrm{H}]^{-}$ion and in the fragment ions whenever this group is still present, it seems worthwhile to determine if any other functional groups of the conjugated bile acid have undergone deprotonation during anion formation or if the negative charge migrates to another part of the fragment during the fragmentation process. It is also interesting to determine if the $[\mathrm{M}-\mathrm{H}]^{-}$ion and the fragment ions do not undergo any rearrangement process other than the migration of hydrogen atoms. To make these determinations, labeling and tandem mass spectrometry experiments have been undertaken.

First, several deuterium-labeled tauroconjugated bile acids were prepared by deuterium exchange or by 

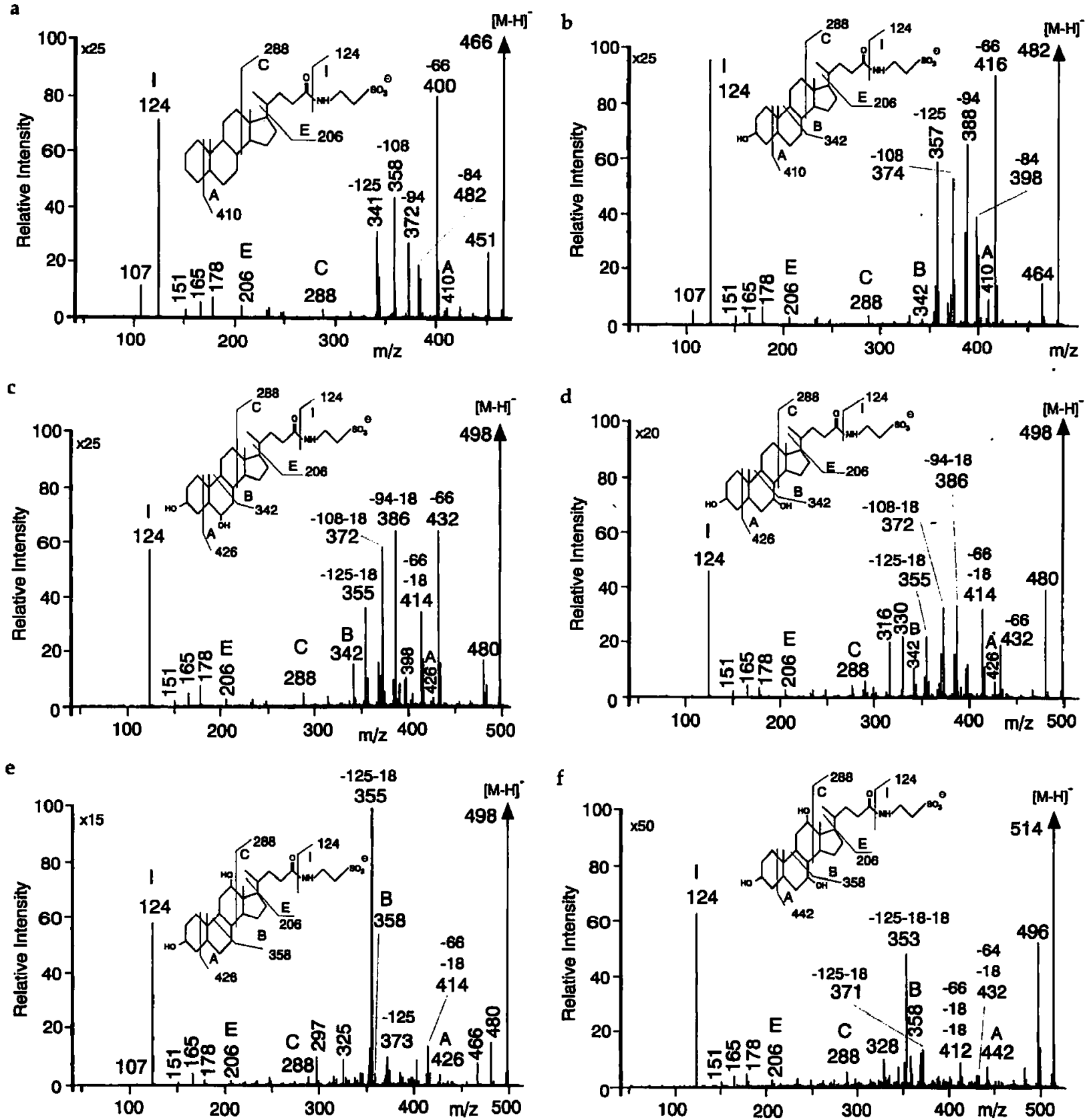

Figure 2. CID fragment spectra of the pseudomolecular anions $[\mathrm{M}-\mathrm{H}]^{-}$at low collision energy (collision offset voltage of $10 \mathrm{~V}$ ) of (a) taurocholanic acid (1), (b) taurolithocholic acid (2), (c) taurohyodeoxycholic acid (4), (d) taurochenodeoxycholic acid (6), (e) taurodeoxycholic acid (8), and (f) taurocholic acid (9).

reduction of keto groups with sodium borodeuteride. By deuterium exchange the compounds are labeled at hydroxyl and amide groups whereas by borodeuteride reduction of keto groups the compounds are labeled on the carbon atoms of the reduced carbonyls. The masses of the fragment ions that contain the sulfonate group in the low energy CID spectra of these two types of deuterium-labeled compounds demonstrate that the deuterium atoms remain in their initial positions. Deuterium scrambling or migration is never observed. For example, after labeling by deuterium exchange of the taurocholic acid (9) to give the tauro- cholic- $d_{4}$ acid $\left(9-\mathbf{d}_{4}\right)$, the ions at $m / z 124$ and 288 are completely shifted to 125 and 289 whereas the ion at $m / z 442$ is shifted to 445 . The taurocholic-3,7,12- $d_{3}$ acid, prepared by reduction with deuteride, displays the unshifted ions at $m / z 124$ and 288 , whereas the ion at $m / z 442$ is completely shifted to 444 .

Second, the low energy CID fragment spectra of all of the ions that contain the sulfonate group have been acquired (Table 4). All of the low energy spectra of the ions $\mathrm{A}$ to $\mathrm{E}$ (Scheme II) display the taurine anion $\left(\mathrm{NH}_{2}-\mathrm{CH}_{2}-\mathrm{CH}_{2}-\mathrm{SO}_{3}^{-}\right)$at $\mathrm{m} / z 124$, and those of the ions $\mathrm{C}$ to $\mathrm{J}$ (Scheme II) display the sulfonate radical 
Table 4. Tabulated fragment spectra of the charge-remote fragmentation of the taurodehydrocholic acid (11). These data demonstrate that the CRF ions are not rearranged; instead, they retain the original structure of the compound

\begin{tabular}{|c|c|c|c|c|c|c|c|c|c|c|c|c|}
\hline \multirow{2}{*}{$\begin{array}{l}\text { Precursor } \\
\text { ions } \\
{[\mathrm{M}-\mathrm{H}]^{-}}\end{array}$} & \multicolumn{12}{|c|}{ Relative intensities of the fragment ions } \\
\hline & 288 & 248 & 234 & 206 & 178 & 165 & 151 & 124 & 107 & 94 & 80 & Others $^{a}$ \\
\hline $438^{b}$ & 8 & 6 & 5 & 5 & 8 & 8 & 2 & 13 & 3 & - & - & $\begin{array}{l}438(100), 436(3) \\
420(5), 232(4), 147(3)\end{array}$ \\
\hline $356^{c}$ & 2 & 4 & 7 & $\cdot 11$ & 12 & 6 & - & 20 & 17 & - & 3 & $\begin{array}{l}356(100), 328(3) \\
324(4), 246(3), 232(4)\end{array}$ \\
\hline 288 & 100 & - & - & 3 & 2 & 5 & 1 & 6 & 3 & - & 6 & $286(3), 222(3)$ \\
\hline 248 & - & 100 & 一 & 2 & 4 & 2 & - & 16 & 10 & - & 12 & $233(6), 182(3)$ \\
\hline 234 & - & - & 100 & 2 & 7 & 1 & 2 & 19 & 14 & - & 20 & - \\
\hline 206 & - & - & - & 80 & - & - & - & 100 & 20 & - & 47 & $142(3)$ \\
\hline 178 & - & - & - & - & 100 & - & - & - & 37 & - & 52 & $\begin{array}{l}70(3), 94(3) \\
112(6), 114(5)\end{array}$ \\
\hline 165 & - & - & - & - & - & 6 & - & - & 39 & 7 & 100 & - \\
\hline 151 & - & - & - & - & - & - & 12 & - & 2 & 94 & 100 & $64(3), 123(10)$ \\
\hline 124 & - & - & - & - & - & - & - & 100 & 5 & 1 & 72 & - \\
\hline 107 & - & - & - & - & - & - & - & - & 83 & - & 100 & - \\
\hline
\end{tabular}

${ }^{a}$ Other peaks present with relative intensities greater than $3 \%$ of the base peak $[\mathrm{m} / \mathrm{z}$ (relative intensity)].

borresponds to cleavage of the $A$ ring.

${ }^{c}$ Corresponds to cleavage of the $B$ ring.

anion $\left(\mathrm{SO}_{3}^{-}\right)$at $m / z$. Table 4 also allows observation of the presence of a fragment at $m / z 288$ from the low energy CID spectra of $[\mathrm{M}-\mathrm{H}]^{-}$, where $A$ and $B$ ions (Scheme II) are precursors. This demonstrates the presence of the intact side chain attached to the $D$ ring in the structure of these higher mass ions.

These two experiments, labeling and tandem mass spectrometry, confirm the position of the negative charge on the sulfonate group during the anion formation. Indeed, deprotonation of another functional group of the conjugated bile acid or a migration of the negative charge would result in migration or scrambling of the deuterium atoms and would not produce the taurine anion or the sulfonate radical anion in the low energy fragment spectra. It is worthwhile to note that no rearrangement (except for some hydrogen migration) of the $[\mathrm{M}-\mathrm{H}]^{-}$ion and of the fragments that contain the sulfonate group could be observed, either by deuterium migration or by the nature of the fragments. Instead, all the data point to a retention of the original structure.

Once the position of the negative charge and the structure of the fragment ions that contain the sulfonate group were determined, attention was focused on the reaction mechanism. Because the negative charge is found to reside on the sulfonate group and because the precursor $[\mathrm{M}-\mathrm{H}]^{-}$ion and the fragment ions that contain the sulfonate group do not rearrange, it may be concluded that the fragmentations that produce higher mass ions (fragments $A$ to $G$, Scheme II) result from remote charge fragmentations.

The ions generated by the charge-remote fragmentation of the steroid rings (fragment ions $A$ to $D$ in Scheme II) can be formed directly from the $[\mathrm{M}-\mathrm{H}]^{-}$ ion or after the introduction of an unsaturation in the steroid nucleus by a preliminary loss of a small neutral, such as hydrogen or water molecules. Indeed, the fragmentation spectra of the $[\mathrm{M}-\mathrm{H}]^{-}$ions contain $[\mathrm{M}-\mathrm{H}-2]^{-}$or $[\mathrm{M}-\mathrm{H}-18]^{-}$ions for every bile acid studied, and the fragmentation spectra of $[\mathrm{M}-\mathrm{H}$ $-2]^{-}$or $[\mathrm{M}-\mathrm{H}-18]^{-}$ions also include these CRF ions (plots not shown). When unsaturations are present in the $A, B$, and $C$ rings, either as keto groups or as endocyclic double bonds, the abundances of these fragment ions (A to D in Scheme II) generally increase. It thus appears that these charge-remote fragments are favored by the introduction of unsaturation in the steroid nucleus.

For example, the fragmentation spectrum of the $[\mathrm{M}-\mathrm{H}]^{-}$ion of taurodehydrocholic acid (11) is shown in Figure 3. This compound displays typical fragments with high abundance at $m / z \quad 438$ and 356, which corresponds to the cleavages of $A$ and $B$ rings. The presence of unsaturations also leads to some hitherto unexplained ions, such as ions at $m / z 436,241,215$, and 147. However, the typical abundant ions are analytically useful.

In addition to this first series (fragments $\mathrm{A}$ to $\mathrm{J}$ in Scheme II), a second series results in contrast from the loss of neutrals from the side chain with charge migration during the fragmentation. It corresponds to the loss of neutrals of $\mathrm{m} / z$ 64 and $66\left(\mathrm{SO}_{2}\right.$ and $\left.\mathrm{H}_{2} \mathrm{SO}_{2}\right), 82$ and $84\left(\mathrm{H}_{2} \mathrm{SO}_{3}\right.$ and $\left.\mathrm{H}_{4} \mathrm{SO}_{3}\right), 94\left(\mathrm{CH}_{2} \mathrm{SO}_{3}\right), 108$ $\left(\mathrm{C}_{2} \mathrm{H}_{4} \mathrm{SO}_{3}\right)$, and $125\left(\mathrm{H}_{2} \mathrm{~N}-\mathrm{CH}_{2}-\mathrm{CH}_{2}-\mathrm{SO}_{3} \mathrm{H}\right)$ as shown in Scheme III and in Figure 2a and $b$. This series dominates the low energy CID spectra of the anions of the tauroconjugated bile acids studied. They are absent from the FAB spectra as well as from the published high energy CID spectra [1, 3]. The loss of the neutrals listed in the foregoing text is clearly borne out by the 


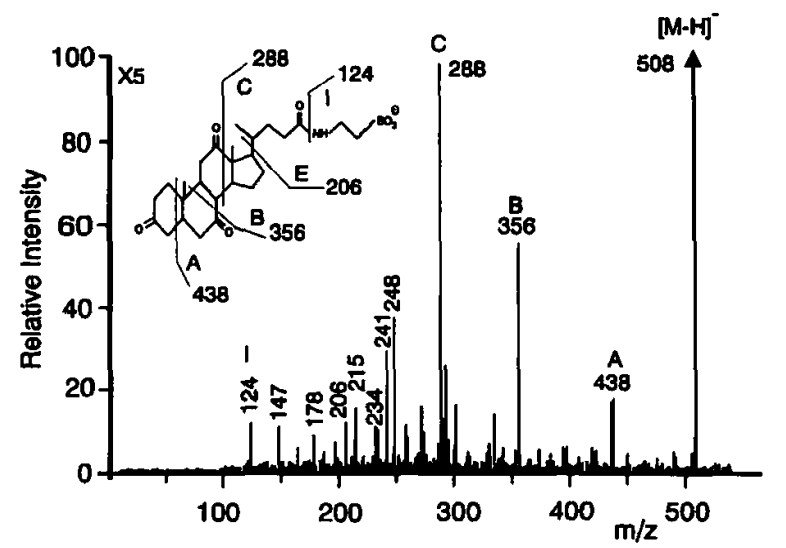

Figure 3. CID fragment spectra of the pseudomolecular anions $\left[\mathrm{M}-\mathrm{H}^{-}\right.$at low collision energy of the taurodehydrocholic acid (11) (collision offset voltage of $10 \mathrm{~V}$ ).

presence of the corresponding ions in all of the spectra of the tauroconjugated acids studied. Loss of $\mathrm{SO}_{2}$ and $\mathrm{SO}_{3}$ as neutral species has already been observed in the gas phase from aromatic sulfonate anions [12] or from sulfonated azo dye anions [13].

Moreover, the presence of hydroxyl groups in the steroid ring system, except for the hydroxyl group at the 3 position, induces loss of water molecules associated with the loss of neutrals shown in Scheme III. No significant differences were detected between the fragmentation spectra of stereoisomers, but the loss of these neutrals permitted distinction of the positional isomers. Indeed, the variation of the numbers or of the positions of the hydroxyl groups changes the relative abundances of these fragments. For example, the taurine conjugates of chenodeoxycholic acid (6) (spectrum in Figure 2d) and of ursodeoxycholic acid (7) (spectrum not shown) display the same fragmentation spectra, whereas the tauroconjugate of deoxycholic acid (8) (spectrum in Figure 2e) displays a very different spectrum. The variations in the relative intensities of the fragments that correspond to the loss of these neutrals,

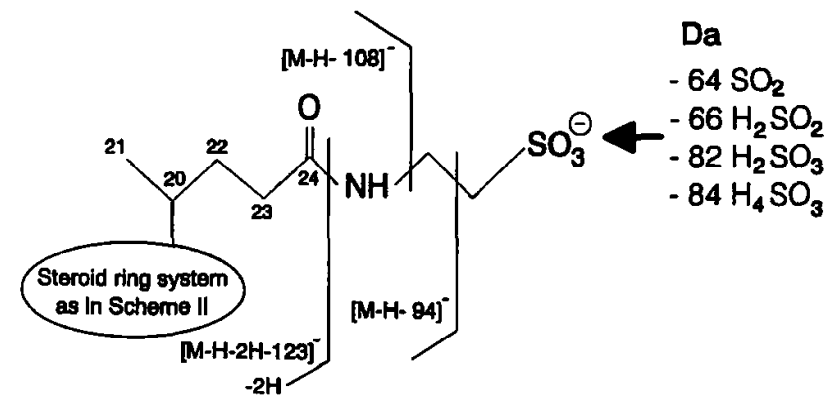

Scheme III. Series of fragment ions produced by the loss of neutrals from the side chain observed in FAB CID tandem mass spectrometry at low energy of the tauroconjugated bile acids.

when structural features are changed in the steroid ring system, are reproducible and are not very sensitive to the CID conditions.

Furthermore, it clearly appears that a hydroxyl group at the 12 position highly favors the loss of water associated with the loss of the taurine moiety, as illustrated in the spectra of taurodeoxycholic acid (8) and taurocholic acid (9) (Figure 2e and f). This is clearly shown in Table 5, which compares the relative abundances of ions that correspond to the loss of taurine with the abundances that correspond to the loss of $\mathrm{H}_{2} \mathrm{SO}_{2}$, another neutral of this series.

This series of fragment ions that occurs by the loss of neutrals from the side chain could be explained as well by mediated charge as by charge-remote mechanisms, if we assume a transfer of the charge, which should then be located on the steroid nucleus rather than on the taurine moiety. However, because taurocholanic acid (1), a bile acid that contains no ionizable group in the steroid nucleus, gives the same fragments, we conclude that the formation of these ions does not involve another charge site located on the steroid nucleus. Thus, these ions result from chargedriven fragmentation reactions.

Table 5. Comparison of the relative intensities of ions that correspond to the loss of taurine with those that correspond to the loss of $\mathrm{H}_{2} \mathrm{SO}_{2}$ for the various tauroconjugated bile acids studied

\begin{tabular}{llll}
\hline \multicolumn{1}{c}{ Bile acids } & \multicolumn{1}{c}{$\begin{array}{c}\text { Relative intensity of } \\
\text { ion that corresponds to } \\
\text { loss of taurine }\end{array}$} & $\begin{array}{c}\text { Relative intensity of } \\
\text { ion that corresponds to } \\
\text { loss of } \mathrm{H}_{2} \mathrm{SO}_{2}{ }^{\mathrm{c}, \mathrm{b}}\end{array}$ & $\begin{array}{c}\text { Ratio of the } \\
\text { two intensities }\end{array}$ \\
\hline \hline Taurocholanic acid (1) & $0.8 \%(341)$ & $2 \%(400)$ & 0.4 \\
Taurolithocholic acid (2) & $1 \%(357)$ & $1.5 \%(416)$ & 0.66 \\
Taurohyodeoxycholic acid (4) & $0.95 \%(355$ and 373$)$ & $1.25 \%(414$ and 432$)$ & 0.76 \\
Taurochenodeoxycholic acid (6) & $1.7 \%(355$ and 373$)$ & $2.5 \%(414$ and 432) & 0.68 \\
Taurodeoxycholic acid (8) & $6.7 \%(355$ and 373) & $0.9 \%(414$ and 432) & 7.4 \\
Taurocholic acid (9) & $1.2 \%(353$ and 371$)$ & $0.27 \%(412$ and 430) & 4.45 \\
Taurohyocholic acid (10) & $1 \%(353$ and 371$)$ & $1.47 \%(412$ and 430) & 0.68 \\
\hline
\end{tabular}

'Sum of the relative intensity of lons that correspond to loss of taurine and ions that correspond to this loss with additional water molecules. The base peak corresponds to the survivor precursor pseudomolecular ion $[\mathrm{M}-\mathrm{H}]$ :

The figures in parentheses correspond to the masses of the resultant fragment ions.

'Sum of the relative intensity of ions that correspond to loss of $\mathrm{H}_{2} \mathrm{SO}_{2}$ and ions that correspond to this loss with additional water molecules. 


\section{Conclusions}

Low collision energy dissociations of tauroconjugated bile acid anions result from two general mechanisms: charge-driven and charge-remote fragmentations. Charge-driven fragmentations dominate in the spectra of saturated ring compounds, and result in the loss of parts of the side chain as neutral species. In mixtures, they permit the recognition of taurine conjugates. Because the relative intensities of some fragments depend on the substituents in the ring, chargedriven fragmentations also yield useful information on positional isomers.

Charge-remote fragmentations are observed at lower relative intensities than in published high energy CID spectra $[1,3]$ or than observed in the FAB spectrum. CRF always appear mixed with charge-driven fragments, which makes observation more difficult, and they are more intense if unsaturations are present in the steroid ring system. In most instances, even at low intensities, $\mathrm{CRF}$ are useful for the determination or the confirmation of the positions of unsaturations or of hydroxy or keto substituents in the rings. For saturated compounds, no backbone rearrangement is observed.

\section{Acknowledgments}

The authors are grateful to A. Spote and R. Rosenberg for their technical help. This work was supported by grant 9.4508.92 from the Belgium National Fund for Scientific Research (FNRS).

\section{References}

1. Tomer, K. B.; Jensen, N. J.; Gross, M. L.; Whitney, J. Biomed. Environ. Mass Spectrom. 1986, 13, 265-272.

2. Tomer, K. B.; Gross, M. L. Biomed. Environ. Mass Spectrom. 1988, 15, 89-98.

3. Griffiths, W. J.; Zhang, J.; Sjovall J. Rapid Commun. Mass Spectrom. 1993, 7, 235-240.

4. Liehr, J. G.; Kingston, E. E.; Beynon, J. H. Biomed. Mass Spectrom. 1985, 12, 95-99.

5. Wood, K. V.; Sun, Y.; Elkin, R. G. Anal. Chem. 1991, 63, 247-250.

6. Van Hoof, F.; Libert, R.; Hermans, D.; Draye, J. P.; Dacremont, G.; de Hoffmann, E. In Adrenoleukodystrophy and Other Peroxisomal Disorders; Uziel, G.; Wanders, R. J.; Cappa, M., Eds.; Excerpta Medica, Elsevier: Amsterdam, 1990; pp 37-60.

7. Libert, R.; Hermans, D.; Draye, J. P.; Van Hoof, F.; Sokal, E.; de Hoffmann, E. Clin. Chem. 1991, 37, 2102-2110.

8. Adams, J. Mass Spectrom. Rev. 1990, 9, 141-186.

9. Gross, M. L. Int. J. Mass Spectrom. Ion Processes 1992, 118/119, 137-165.

10. Wysocki, V. H.; Mark, E. B.; Cooks, R. G. Org. Mass Spectrom. 1988, 23, 627-633.

11. Zhang, J.; Griffiths, W. J.; Bergman, T.; Sjovall, J. J. Lipid Res. 1993, 34, 1895-1900.

12. Binkley, R. W.; Flechtner, T. W.; Tevesz, M. J. S.; Winnik, W.; Zhong, B. Org. Mass Spectrom. 1993, 28, 769-772.

13. Richardson, S. D.; McGuire, J. M.; Thruston, A. D.; Baughman, G. L. Org. Mass Spectrom. 1992, 27, 289-299. 